\title{
Analysis of the survival of patients receiving systemic treatment for melanoma of the skin: a retrospective population study with patients treated in Poland in 2011-2015
}

\author{
Melania Brzozowska ${ }^{1,2}$, Waldemar Wierzba ${ }^{3}$, Andrzej Śliwczyński ${ }^{3,4}$, Gabriela Majkut ${ }^{5}$, Andrzej Jacyna ${ }^{6}$, \\ Ewa Orlewska ${ }^{7}$, Piotr Potemski ${ }^{8}$, Michał Marczak ${ }^{1}$
}

1Division of Quality Services, Procedures and Medical Standards, Medical University of Lodz, Lodz, Poland
2Department of Drug Economics, National Health Fund, Central Office, Warsaw, Poland
3UHE Satellite Campus in Warsaw, University of Humanities and Economics in Lodz, Poland
${ }^{4}$ Department of Healthcare Services, National Health Fund, Central Office, Warsaw, Poland
${ }^{5}$ National Health Fund, Warsaw, Poland
${ }^{6}$ National Health Fund, Central Office, Warsaw, Poland
${ }^{7}$ Faculty of Medicine and Health Sciences, Jan Kochanowski University, Kielce, Poland
${ }^{8}$ Copernicus Memorial Hospital, Comprehensive Cancer Center and Traumatology, Medical University of Lodz, Lodz, Poland

${ }^{8}$ Copernicus Memorial Hospital, Comprehensive Cancer Center and Traumatology, Medical University of Lodz, Lodz, Poland
Adv Dermatol Allergol 2019; XXXVI (4): 438-441

DOI: https://doi.org/10.5114/ada.2019.83368

\begin{abstract}
Introduction: In Poland, it is uniquely possible to assess real effects of the introduction of new oncological therapies on the overall survival in patients as such therapies are funded by one payer only - the National Health Fund (NHF). Data collected by the NHF make it possible to analyse the survival of all patients who were diagnosed with melanoma.

Aim: The paper presents findings of a retrospective analysis of the efficacy of systemic treatment in patients with malignant melanoma of the skin in Poland with regard to the overall survival.

Material and methods: The analysis of the overall survival was performed with the Kaplan-Meier method in the population receiving systemic treatment. Three groups of patients were analysed. Group 1 included all patients who had started systemic treatment between 1 March 2011 and 1 March 2015: 1,258 patients. The median overall survival was 8.4 months. Group 2 included 444 patients who had started systemic treatment between 1 March 2011 and 28 February 2013. The median overall survival was 6.6 months in this group. Group 3 included 814 patients who had started systemic treatment between 1 March 2013 and 1 March 2015 and included 546 patients who were also treated in drug programmes with ipilimumab and vemurafenib (approx. 67\%). The median overall survival was 9.4 months.

Results: A difference in the overall survival between group 3 and 2 was statistically significant $(p<0.05)$.

Conclusions: The introduction of vemurafenib and ipilimumab into systemic treatment in Poland using public funds had a significant effect on the prolongation of the overall survival in patients with malignant melanoma of the skin.

Key words: melanoma treatment, vemurafenib, ipilimumab, Poland.
\end{abstract}

\section{Introduction}

Randomised clinical trials make it possible to assess the efficacy and safety of new therapies, but they are in general different from observations performed in everyday clinical practice. The incidence of coexisting diseases, the age structure of patients, their performance status, the organisation of health care system/organisation of care strategies for patients, all these elements may be different in clinical trials compared to real populations of patients. Therefore, all these affect the overall survival

Address for correspondence: Gabriela Majkut, National Health Fund, 186 Grojecka St, 02-390 Warsaw, Poland, phone: + 48225726405 , e-mail: gabriela.majkut@nfz.gov.pl

Received: 14.03.2018, accepted: 1.05.2018. 
levels observed. It is more and more difficult to assess the overall survival (OS) and disease-specific survival (DSS) in clinical trials as the follow-up period has to be sufficiently long, a group of patients in the study should be large enough, and there has to be a possibility of treatment after discontinuation of a clinical trial. Therefore, such endpoints are often replaced with intermediate endpoints such as progression-free survival (PFS), but they introduce elements of a subjective assessment of disease progression by a physician or a patient.

In Poland, it is uniquely possible to assess real effects of the introduction of new oncological therapies on the overall survival in patients as such therapies are funded by one payer only - the National Health Fund (NHF). Data collected by the NHF make it possible to analyse the survival of all patients who were diagnosed with melanoma. Funds for new therapies are secured in drug programmes as such programmes are a separate form of financing. In Poland, until 1 March 2013, the systemic treatment funded from public funds for patients with melanoma was limited to chemotherapy and immunotherapy with interferon. Since 1 March 2013 the following medications have been gradually included into public funds: vemurafenib (funded according to the decision of the Minister of Health since 1 March 2013); ipilimumab (funded according to a decision of the Minister of Health since 1 March 2014).

\section{Material and methods}

Using NHF report databases data from 2011-2015, regarding patients with the diagnosis of C43 (ICD-10 code) - malignant melanoma of the skin, who received systemic treatment were collected for analysis. Using the unique patient identifiers (patient IDs such as PESEL numbers), the following associated information was collected: sex, date of birth, as these data were reported by service providers. The analysis of the overall survival was performed with the Kaplan-Meier method in the population receiving systemic treatment (chemotherapy and/or medications administered in drug programmes). The start of the observation was assumed as the date of the first administration of systemic treatment (the first date of administration of chemotherapy or medication as part of a drug programme). The data regarding deaths were taken from the PESEL database managed by the Ministry of Internal Affairs in Poland. The overall survival was calculated for the whole study population and two subgroups of patients defined as follows: a) patients who had started systemic treatment in the period from $01 / 03 / 2011$ to $28 / 02 / 2013$, and b) patients who had started systemic treatment in the period from 01/03/2013 to 01/03/2015). 31 December 2015 was the cut-off point for data associated with survival in all analyses. The SAS E.G. ver. 5.1 was used to prepare the results of analyses. A difference in the overall survival was estimated with a two-sided log-rank test at the statistical significance of $p<0.5$.

\section{Results}

\section{Group 1}

The analysed population included all patients who had started systemic treatment between 1 March 2011 and 1 March 2015: 1258 patients (53.3\% of men and $46.7 \%$ of women). The age of men in the analysed population ranged between 4 and 86 years; the median age was 52 years (mean: 51.7, standard deviation: 20.57). The age of women in the analysed population ranged between 18 and 92 years; the median age was 54.5 years (mean: 54.4, standard deviation: 20.30). The median follow-up for the whole population was 29.93 months.

Figure 1 presents the Kaplan-Meier curve for OS in this group.

The number of censored observations was 244 (19.4\%). The median overall survival was 8.4 months. The 12-month survival probability was 37.4\%, 24-month survival probability - 21.5\%, 36-month survival probability $-15.1 \%$, and 60 -month survival probability $-11.1 \%$.

\section{Groups 2 and 3}

In the period between 1 March 2011 and 28 February 2013 (group 2), 444 patients had started systemic treatment and between 1 March 2013 and 1 March 2015 (group 3), 814 patients had started treatment, and this group included 546 patients who were also treated in drug programmes (approx. 67\%).

Group 2 included $51.1 \%$ of men and $48.9 \%$ of women; the median age of men was 54.5 years (mean: 54.0, standard deviation: 17.79), and the median age of women was 54.5 years (mean: 53.3, standard deviation: 17.85).

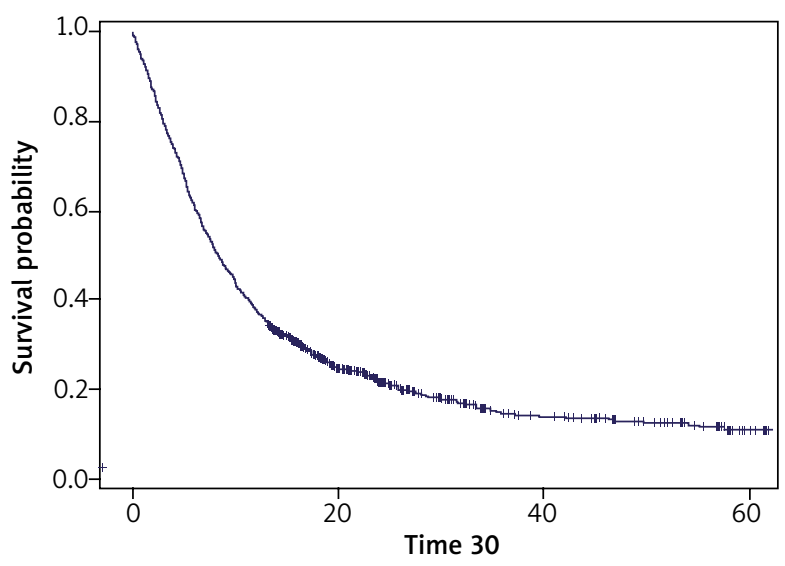

Figure 1. The Kaplan-Meier curve for group 1 (patients who had started systemic treatment between 1 March 2011 and 1 March 2015) 


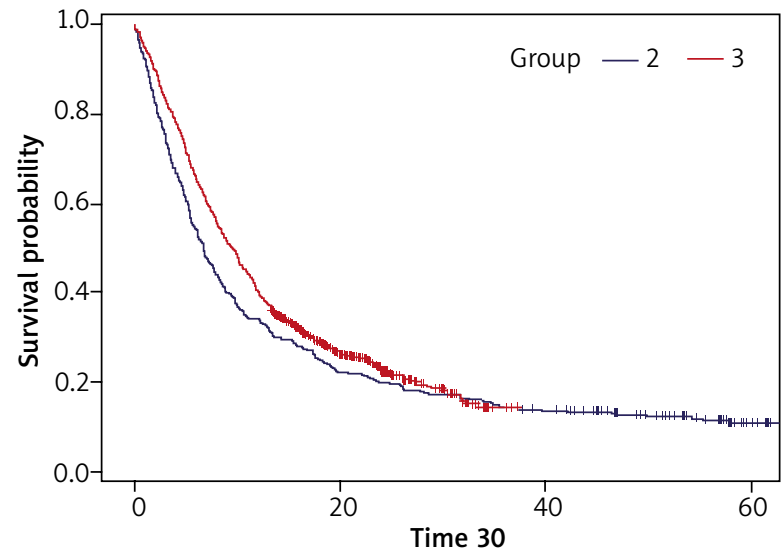

Figure 2. The Kaplan-Meier curves for groups 2 and 3 (patients who had started systemic treatment between 1 March 2011 and 28 February 2013 and between 1 March 2013 and 1 March 2015)

Group 3 included $54.3 \%$ of men and $45.7 \%$ of women; the median age of men was 57 years (mean: 56.3 , standard deviation: 12.30), and the median age of women was 61 years (mean: 59.7, standard deviation: 11.93).

Figure 2 presents Kaplan-Meier curves for OS in these groups.

In group 2, the number of censored observations was 53 (11.94\%). The median follow-up for group 2 was 53.77 months. The median overall survival was 6.6 months. The 12-month survival probability was 33.8\%, 24-month survival probability - 19.8\%, 36-month survival probability $-14.4 \%$, and 60 -month survival probability $-10.5 \%$.

In group 3, the number of censored observations was 191 (23.46\%). The median follow-up for group 3 was 24.03 months. The median overall survival was 9.4 months. The 12 -month survival probability was $39.3 \%$, 24-month survival probability - 22.4\%, 36-month survival probability $-14.4 \%$, and 60 -month survival probability $14.7 \%$. A difference between the overall survival of group 3 and 2 was statistically significant $(p=0.013)$.

\section{Discussion}

In Poland patients who had started systemic treatment for melanoma between 1 March 2013 and 1 March 2015 had significantly longer overall survival compared to those who had started such treatment between 1 March 2011 and 28 February 2013. The median OS increased from 6.6 to 9.4 months and the 1-year survival rate from $34 \%$ to $39 \%$. A difference in the survival rates was visible for the first 2 years of the follow-up, in the later period the survival curves overlapped and the 2- and 3-yearsurvival rates were similar.

In our opinion, based on the analysed group, it is possible to conclude regarding the real effects of new medications on improved prognosis in the whole coun- try. According to the data from the National Cancer Registry, approximately 1400 patients die from melanoma in Poland every year [1]. Taking into account that for medical and organisational reasons some patients do not receive any systemic treatment, some approximately 400 patients who start treatment annually after 2013 seem to be representative. In the earlier period, the number of patients receiving systemic treatment was lower (more than 200 patients annually), and it might have been associated with the fact that physicians thought, but without any evidence, that patients might have received benefits from standard chemotherapy.

In our opinion, the introduction of ipilimumab and vemurafenib funded by public funds was the most probable cause why the OS was longer in patients starting the systemic treatment in the later period. As many as $2 / 3$ patients in group 3 had been receiving ipilimumab or vemurafenib as part of a drug programme for patients with metastatic and inoperable melanoma. Drug programmes have relatively strict eligibility criteria including, among others, appropriate values of laboratory parameters and the performance status. These criteria are less restrictive compared to those applied in clinical studies, but patients have to be selected appropriately. This financing system for new medications reduces the risk of the significantly lower efficacy of new medications in real life compared to that demonstrated in clinical trials. However, it has the obvious limitations.

In July 2011, using the results of the phase III study, the European Medicines Agency approved ipilimumab. In patients with metastatic or inoperable melanoma who had been receiving previous systemic treatment, this drug increased the median OS compared to the control group from 6.4 to 10.1 months and increased the 1-year survival rate from $25 \%$ to $46 \%$, and the 2 -year survival rate from $14 \%$ to $24 \%$ [2]. Patients who are treated with ipilimumab outside clinical trials may, however, experience significantly lower benefits of treatment. And in fact, in 193 patients in the Expanded Access Programme in the UK, the median OS was only 6.1 months, and the 2-year survival rate was 14\% [3]. In the EU countries, vemurafenib was approved for marketing in February 2012 based on the findings of the phase III study where the median OS was 13.6 months in patients with a V600 $B R A F$ mutation who had not received previous systemic treatment $[4,5]$.

Patients who were included in a drug programme conducted in Poland since 1 March 2015 were receiving ipilimumab and vemurafenib only as second-line treatment. Moreover, 1 patient was allowed to take only one of these agents. This factor might have some effects on impairment of survival parameters in patients receiving vemurafenib compared to the findings from a registration study where this agent was used as first-line treatment. In a large group of more than 3 thousand patients who participated in a study assessing mainly the safety 
of treatment with vemurafenib in a population similar to the one observed in clinical practice, the median OS was 12 months and the rate of patients who were still alive after 12 and 18 months was 52\% and 36\%, respectively [6]. Half of these patients had been receiving systemic treatment in the past.

It is not surprising to see separate survival curves of groups 2 and 3 during the first 2 years of follow-up only. It can be suspected that OS improvement depended mainly on the effects of vemurafenib treatment and this agent had been already funded one year before ipilimumab. Moreover, ipilimumab is an agent with relatively low activity in a short-term follow-up, however, in as many as $22 \%$ of patients treated in phase 2 and 3 clinical trials, it allowed to achieve 3-year and longer survival [7]. Maybe if patients from group 3 had been observed longer, we might have been able to demonstrate such effects. Of course, evident limitations of our analysis include lack of detailed information on the stage of advancement, prognostic factors, response-to-treatment rate, and progression-free survival. It is a result of the fact that we included all patients starting systemic treatment (funded by public funds) due to melanoma in our country within 4 years. We cannot exclude a possibility that a low number of patients, especially at an early stage, had been receiving systemic treatment as adjuvant treatment despite the fact that such a strategy is not medically justified. However, it might have had a hypothetical effect on improved survival in the early period contrary to the late period, and it cannot be used to explain a difference in the prognosis that was observed.

\section{Conclusions}

The introduction of vemurafenib and ipilimumab into systemic treatment in March 2013 in Poland using public funds had a significant effect on the longer overall survival in patients with malignant melanoma of the skin.

\section{Conflict of interest}

The authors declare no conflict of interest.

\section{References}

1. Wojciechowska U, Didkowska J. Zachorowania i zgony na nowotwory złośliwe w Polsce. Krajowy Rejestr Nowotworów, Centrum Onkologii - Instytut im. Marii Skłodowskiej-Curie. Web address: http://onkologia.org.pl/raporty/, access 19.11.2016.

2. Hodi FS, O’Day SJ, Mc Dermott DF, et al. Improved survival with ipilimumab in patients with metastatic melanoma. N Engl J Med 2010; 363: 711-23.

3. Ahmad SS, Qian W, Ellis S, et al. Ipilimumab in the real world: the UK expanded access programme experience in previously treated advanced melanoma patients. Melanoma Res 2015; 25: 432-42.
4. Chapman PB, Hauschild A, Robert C, et al. Improved survival with vemurafenib in melanoma with BRAF V600E mutation. N Engl J Med 2011; 364: 2507-16.

5. McArthur GA, Chapman PB, Robert C, et al. Safety and efficacy of vemurafenib in BRAF(V600E) and BRAF(V600K) mutation-positive melanoma (BRIM-3): extended follow-up of a phase 3, randomised, open-label study. Lancet Oncol 2014; 15: 323-32.

6. Larkin J, Del Vecchio M, Ascierto PA, et al. Vemurafenib in patients with BRAF(V600) mutated metastatic melanoma: an open-label, multicentre, safety study. Lancet Oncol 2014; 15: 436-44.

7. Schadendorf D, Hodi FS, Robert C, et al. Pooled analysis of long-term survival data from phase II and phase III trials of ipilimumab in unresectable or metastatic melanoma. J Clin Oncol 2015; 33: 1889-94. 